\title{
Terminologie im Sprachbuch aus der sicht der schulbucharbeit
}

Wenn man ein schulbuch macht, dann kommt irgendwann im Laufe der Arbeit die Frage nach der Terminologie auf, in unserem Falle im Bereich Grammatik. Die Entscheidung darüber ist nicht ganz frei, denn es gibt Vorgaben in den Lehrplänen der Bundesländer, Vorkenntnisse und Vorhaben von Lehrern, Besonderheiten einzelner Sprachen, so daß Autoren und Verlagsredaktionen sich aufeinander und auf die genannten punkte abstimmen müssen, wie ich im 2 . Teil meines Artikels ausführen will. Daher ist es im Prinzip zu begrüßen, wenn eine Ubersicht über die auflistbaren Daten erstellt wird, die in eine Empfehlung münden kann. Ob allerdings die terminologischen Empfehlungen der Konferenz der Kultusminister die Erfüllung eines Desiderats darstellen, darf man bezweifeln.

\section{Zum Hintergrund der KMK-Empfehlungen}

Die KMK-Empfehlung grammatischer Termini (KMK-Liste) ist nicht der einzige Versuch der letzten Jahre, Terminologie zu vereinheitlichen. Allein in stuttgart (womöglich auch anderswo) sind zwischen 1978 und 1980 zwei Listen mit derselben zielsetzung erstellt worden, nämlich der "Entwurf einer Basisliste grammatischer Fachausdrücke" der Landesstelle für Erziehung und Unterricht in Stuttgart vom März 1980 (Basisliste LEU) sowie ein internes Klett-Papier, die "Empfehlung zur Verwendung grammatischer Bezeichnungen in den Sprachbüchern des Ernst Klett Verlages" 2. Aufl. Juli 1980, an der die Redaktionen Deutsch/alte Sprachen, moderne Fremdsprachen, Deutsch für Ausländer und Grundschule beteiligt waren (Klett-Liste). Schon auf den ersten Blick stellte sich in Aufbau und Terminologie eine so große Ähnlichkeit zwischen KMK-Liste und Klett-Liste heraus (auBer an den Stellen, an denen die KMK-Liste besonders angreifbar ist; ich nenne sie später), daß es mir lohnend schien, der Entstehung der Listen einmal nachzugehen, soweit ich das in der kurzen zeit konnte. 
Das Ministerium für kultus und sport in stuttgart (MKS) beauftragte im Herbst 1977 die LEU, "einen Arbeitskreis "Abstimmung der grammatischen Terminologie" einzurichten. Die Mitglieder wurden aus dem Bereich der Hauptschule, der Realschule und des Gymnasiums berufen." (Basisliste LEU 1980, S. 1). Die erste Fassung der Basisliste erschien im Mai 1978, dann erfolgte eine Uberarbeitung nach den damals neuen Lehrplänen in Baden-Württemberg, bis die Fassung vom März 1980 herauskam.

Diese Basisliste ist recht umfangreich. AuBer einer neunseitigen Einführung enthält sie auf dreiBig Seiten die Auflistung grammatischer Fachausdrücke für das Fach Deutsch mit Erläuterungsspalte und eine Spalte für Entsprechungen in den Fächern Englisch, Französisch, Latein, ferner elf Seiten synopse der Tempora der verschiedenen sprachen. Während eine solche Liste als Ubersicht den Schulbuchmachern schon von Nutzen sein kann, sollte m.E. die Bezeichnung "Basisliste" keinesfalls so verstanden werden, das hier etwa terminologisches Basiswissen versammelt wäre. Dazu ist die Liste bei weitem zu umfangreich, und dazu ist der theoretische status vieler Termini auch zu anfechtbar. Ich nenne die "Pronominaladverbien" mit den Untergruppen "Demonstrativadverb, Frageadverb, Relativadverb"; ich nenne das "Prädikatsnomen" im Unterschied zum "Prädikativum"; ich nenne das "restringierende, dramatische bzw. historische Präsens". DaB die Basisliste LEU so aussieht, könnte man auch anhand ihrer Mitarbeiterliste personalisieren, was ich hier nicht tun will. Jedenfalls spiegelt sie eher den stand der sprachdidaktik, wie er in der Blütezeit der Linguistisierung war. Vielleicht hätte eine solche Basisliste zehn Jahre früher noch Begeisterung erregt.

Nun zu der verlagsinternen Klett-Liste. Während ihrer Arbeit wande sich die LEU-Kommission an den Klett-Verlag mit der Frage, ob es hier nicht eine Terminologie-Empfehlung gebe. Das war der entscheidende AnstoB, ein schon länger ins Auge gefabtes vorhaben nun endlich $\mathrm{zu}$ realisieren: Eine erste Liste wurde von Redakteuren der beteiligten Redaktionen 1979 zusammengestellt, die 2. (hektografierte) Auflage erfolgte 1980. Diese Liste ist 
viel kürzer als die Basisliste LEU, sie umfaBt nur vier Seiten kleineren Schreibmaschinentyps. Der Grund für den Unterschied kann darin liegen, daB inzwischen die Linguistisierungs- und Terminologiewelle nicht nur abgeebbt, sondern auch heftiger kritik ausgesetzt gewesen war, wovon auch das klett-sprachbuch $1970 \mathrm{ff}$. betroffen war.

Theorie- oder terminilastige sprachbücher hatten weniger Marktanteile, worauf der klett-Verlag mit einer Uberarbeitung und straffung der Grammatikteile seines sprachbuchs reagierte. Das erklärt auch, warum die in dieser situation entstandene KlettListe mit den Termini sparsamer umgeht. Diese Klett-Liste ging an die LEU, soviel ist sicher. Irgendwie ist dann eine ziemliche Ähnlichkeit der KMK-Liste 1982 mit der Klett-Liste 1980 entstanden, bis auf die erwähnten geringen, aber wichtigen Abweichungen, die noch $z u$ besprechen sind.

Falls es tatsächlich so wäre - was ich nicht weiB -, daß die KMK-Liste eine von Theorie befreite Klett-Liste wäre, so müBte man erst recht fragen, welche sprachwissenschaftler und -didaktiker an ihr mitgewirkt haben. Denn diese bleiben anonym; bei der Basisliste LEU werden die Mitarbeiter benannt.

\section{Ver lagsimage}

Leider konnte ich nicht in Erfahrung bringen, ob auch andere Verlage bis $z u$ Terminologielisten gelangt sind. Als Schulbuchautor erfährt man aber immer wieder, dab Verlage versuchen, ihr programm abzustimmen. Welche internen Interessen gibt es dafür?

Das vordergründigste Interesse möchte ich Verlagsimage nennen. Das bedeutet, das ein verlag nicht nur ein einzelnes produkt verkauft, sondern das produkt als Teil eines sortiments, fir das er durch seinen Namen eine gewisse Qualitätsgarantie übernimmt. Von einem Verlag wird möglicherweise erwartet, daB sein Image einigermaßen einheitlich bleibt bzw. daß inhaltliche Abstimmungen zwischen den einzelnen produkten vorgenommen sind, am einfachsten wohl in terminologischen Fragen. Da Terminologie fach- 
liche Verständigung ist, muß man es als sinnvoll ansehen, zwischen einzelnen schulfächern, die ein Verlag bedient, solche Regelungen zu treffen, schon damit die terminologische Kapazität der schüler nicht überbelastet wird. Ob das sinnvoll möglich ist, muB ein Vergleich der einzelnen Schulsprachen ergeben. zwar wird selten vorkommen, $d a \beta$ an schulen nur produkte eines Verlags für alle schulsprachen angeschafft werden. Da aber viele Lehrer zwei sprachen unterrichten, z.B. Deutsch und Französisch, kann die Abstimmung des Verlagsprogramms nach guten Erfahrungen mit dem einen Werk die Anschaffung eines $z$ weiten Werks aus demselben Verlag begünstigen.

3. Schüler und Lehrer

Es ist schon erwähnt worden, auf wen schulbücher zielen, auf Schüler und Lehrer nämlich, und es gibt gute Gründe anzunehmen, daB sie sich weitaus stärker am Lehrer orientieren als am Schüler. Denn Verlag und Autoren wissen über Schüler einfach weitaus weniger: Was als Rückmeldung aus Erprobungen von sprachbucheinheiten oder als Kommentar zu fertigen sprachbüchern kommt, kommt von Lehrern oder ist zumindest von Lehrern angeregt.

Auch der Schulbuchmarkt spiegelt die Wertschätzung eines Buches durch Lehrer, kaum die durch schüler. Was wir wissen ist, daB Schüler grammatische Termini entweder erst gar nicht richtig bilden oder verwechseln und vergessen, je mehr, desto mehr, im Sinne der Regel: The more you know, the more you forget, why study? Das ist schon ein Argument dafür, die Terminologie in Sprachbüchern gering $z u$ halten zugunsten des operierens an der Sprache. Die wirklich wichtigen Termini (welche sind das? Zweifellos nicht die, die nur in einem Jahrgang gebraucht werden und dann nie mehr, auch nicht in den kommunikativen Teilen desselben Sprachbuchs!) sollten dann unter allen sprachlichen Fächern möglichst gut abgestimmt werden, damit durch die verschiedenen Schulsprachen eine gewisse konditionierende wiederholung der betreffenden Termini stattfindet. Soweit eine hehre Forderung, die im 4. Abschnitt noch problematisiert wird. 
Auberdem, um bei den postulaten zu bleiben, sollte Terminologie schulartspezifisch benutzt werden. Das heiBt in den Lehrplänen einerseits, daß mengenmäBig unterschieden wird, indem z.B. das Objekt im Genitiv der Realschule und dem Gymnasium vorbehalten wird, andererseits werden in manchen Lehrplänen für Grund- und Hauptschule deutsche Termini, für die "höheren" Schulen jedoch lateinische vorgeschrieben.

Glücklicherweise sind die Zeiten vorbei, da die schüler fundamental von sprachbuch zu sprachbuch umlernen musten, zum Beispiel von Nominalphrase zu Substantivgruppe (also nicht nur terminologisch!). Glücklicherweise sind diese zeiten auch für die Lehrer vorbei. Lehrererwartungen an sprachbücher allgemein kennen wir vor allem aus den Berichten des AuBendienstes der Verlage, der ja den Kontakt zwischen Redaktion und schule symbolisiert:

- eine theoretische Grundlage und Terminologie, die ein nicht besonders fortgebildeter Lehrer versteht,

- methodischen Einfallsreichtum,

- wenig Aktivitäten außerhalb des Klassenzimmers wie Erkundungen oder Materialbeschaffung ...

In terminologischer Hinsicht haben Lehrer manchmal Schwierigkeiten mit Sprachbüchern, auf die Verlage sich einstellen müssen, wenn sie Sprachbïcher absetzen wollen:

- Segmentierung der grammatischen Phänomene in zu viele Untergruppen führt zu Verwirrung (übrigens nicht nur der Lehrer). Beispiel Attribute: Adjektiv-Atrribut, Genitiv-Attribut, Präpositionalattribut, Adverb-Attribut, Infinitiv-Attribut, Attributsatz, Apposition. Beispiel Adverb wie eingangs zitiert, Pronominaladverbien sind Relativadverb, Demonstrativadverb, Frageadverb.

- Neueinführung von grammatischen Termini, die schon anders besetzt sind, schafft Verwirrung. Als Beispiel soll der weite Prädikatsbegriff dienen (Verb und Ergänzungen), der dazu führte, daB der Begriff "Prädikat" vielfach ganz abgelehnt wurde. 
Auch bei der Satzfunktion wurde darum in Deutsch einfach von "Verb" gesprochen wie im Englischen.

- Umlernen ist am schwierigsten auch für Lehrer, und dies besonders bei Denkmodellen und Termini. Umlernen ist z.B. nötig, auch nach der KMK-Liste, bei Adjektiv und Adverb als Wortart und bei ihrer satzfunktion als Attribut oder Adverbial. Im Deutschen bleibt nach unseren Sprachbüchern ein Adjektiv immer der Wortart Adjektiv zugehörig, ganz gleich, ob es als Adverbial, Attribut, Prädikativum verwendet wird (Das Auto ist schnell. Das schnelle Auto. Das Auto fährt schnell.). Die Zuordnung wortart - Satzfunktion kann in anderen Sprachen anders geregelt sein, ohne daß sich dadurch für das Deutsche etwas ändert, in den anderen schulsprachen zum Beispiel durch Adverb-Ableitung aus dem Adjektiv (im Englischen offenbar mit Tendenz, das Adjektiv beizubehalten), die dann als Adverbial gebraucht werden kann: rapide - rapidement / vite; quick quickly / fast. Noch schwieriger ist das Umlernen, wenn man objekte/Ergänzungen und Adverbiale auseinanderhalten will. Nicht umsonst scheitert hier sogar die KMK-Liste, die an dieser stelle leider von der klett-Liste abweicht.

In der Klett-Liste wird - in Ubereinstimmung z.B. mit den KlettSprachbüchern, die hier einen dependenziellen Ansatz vorstellen - die präpositionalergänzung konsequent als eine von der verbsemantik geforderte Leerstelle angesetzt, während Adverbiale zusätzliche satzglieder sind. Da sich die KMK-Liste laut Vorwort aber keinem theoretischen Ansatz verpflichten mag und infolgedessen die traditionelle Grammatik (listigerweise) für keinen solchen hält, ist ihr eigentlich der Boden für die Unterscheidung von Präpositionalergänzung/Präpositionalobjekt und Adverbiale entzogen. Dennoch geistern ja beide Termini herum, und das Fehlen eines der beiden würde einer Begründung bedürfen. Daher empfiehlt KMK die Unterscheidung der beiden aus "unterrichtspraktischen Gründen". Ich frage mich, was an dieser - übrigens als "schwierig" bezeichneten - Abgrenzung praktisch sein soll. Viele Lehrer, die ich kenne, neigen dazu, alle präpositionalen Substantivgruppen als Adverbiale zu klassifizieren, und das ist 
wirklich praktisch. Oder sollte es doch Unterschiede in der Sprache geben?

Wir stoßen hier an die Grenze der rein terminologischen Argumentation: Terminologie braucht eine theoretische Basis, die auch Methoden bereitstellt, nach denen dann von Lehrern Klassifizierungen vorgenommen werden können, in unserem Beispiel die Verbsemantik.

DaB dergleichen solche Schwierigkeiten macht, 1 iegt in der Lerngeschichte all der Lehrer begründet, die entweder in ihrer Ausbildung von deutscher Grammatik verschont geblieben sind oder die als schüler einen ungeliebten und unreflektierten Grammatikunterricht genossen haben. Als Deutschlehrer müssen sie nun alte Kenntnisse aus der Latein-oder sonstigen Fremdsprachengrammatik reaktivieren, sofern sie nicht eine Fremdsprache auch noch studiert haben. So reproduziert sich die Lerngeschichte der Lehrer über die Schüler, die wieder Lehrer werden.

4. Vergleichbarkeit der Unterrichtssprachen

Ein wirklich heikler Punkt ist die Vereinbarkeit der für jede einzelne schulsprache angemessenen Terminologie mit der jeweils anderen. Der Konsens über terminologische Fragen, den beispielsweise die Klett-Liste repräsentiert, ist nicht mehr erkennbar als ein dünner und an verschiedenen stellen problematischer, zu dem einige oder alle Redaktionen sich vielleicht nur halbherzig bereitgefunden haben. Ein Grund für die Halbherzigkeit ist natürlich, daß für die einzelnen sprachen am liebsten Bezeichnungen in der jeweiligen sprache gewählt werden. Weiter scheint es doch so zu sein, daß nicht alle Grammatikmodelle für alle Sprachen in gleicher Weise angemessen sind, trotz der von Linguisten oft beschworenen Universalität ihrer Modelle. Man denke nur an den aus der gestuften Dependenz entwickelten weiten Prädikatsbegriff, der für Anglisten offenbar kaum akzeptabel ist, an das Kasussystem oder an das Tempussystem. Ähnliche Schwierigkeiten machen wieder Ergänzung und Angabe bzw. Adverbial durch die verschiedenen sprachen. 
Um hier überhaupt zu einer Vergleichbarkeit zu kommen, mübte zwischen den einzelnen schulfächern geklärt werden, ob es eine nicht nur terminologische, sondern auch theoretische Basis geben kann, da ja theorieunabhängige Terminologie nicht existiert. Wer kann so etwas klären? Die KMK hat mit ihrer vermeintlich theoriefreien Terminologieliste nicht überzeugt. Besser wäre es schon, eine Gruppe von Linguisten und sprachdidaktikern nähme das in die Hand, und warum nicht das IdS? Den mutter- und fremdsprachlichen Verlagsredaktionen und ihren zahlreichen Autoren obläge dann immer noch die Aufgabe, ihre Vorstellungen von Fachspezifik und diaktischer Grammatik zu koordinieren und an der erarbeiteten Empfehlung zu orientieren. Natürlich stünde es ihnen auch frei, von der Empfehlung abzuweichen. Empfehlung heibt ja nicht Reglementierung.

\section{Lehrpläne der Bundesländer und Verlagsarbeit}

Es wird mir ganz unwohl bei dem Gedanken, daß eine an sich wünschenswerte Theorie der Schulsprachen in den Lehrplänen der Bundesländer festgeschrieben wird. Leider ist das Festschreiben von Theorien in Baden-Württemberg schon Tradition. Die Lehrpläne ab 1977 verpflichteten die schulen auf eine modifizierte Dependenzgrammatik mit Ersatzproben statt W-Fragen. Der ganz neue Lehrplan unter dem neuen Kultusminister hingegen (Lehrplanrevision in Baden-Württemberg 1983/84) bekannte sich zur traditionellen Grammatik, deren Termini er verbindlich vorschreibt. Beides ist m.E. nicht die Aufgabe von Lehrplänen. Kehren wir zur rein terminologischen Frage zurück: Der neue Lehrplan von Baden-Württemberg $1983 / 84$ geht in den "grundsätzlich verbindlichen grammatikalischen Begriffen" schon für die Realschule über die KMK-Liste hinaus, z.B. mit "Konjunktionalsatz, Apposition, Partizip Präsens und Partizip Perfekt, SatzschluBzeichen". Er legt eindeutig den Begriff "Substantiv" gegenüber "Nomen" fest usw. Was heibt das für die schulbuchverlage? Vorbei sind die zeiten, in denen Verlage das munter ignorieren konnten. Wie die schulaufsicht insgesamt, so ist auch die schulbuchzulassung strikter geworden, wobei terminologische 
Setzungen ein wichtiger Punkt sind. Da überhaupt weniger Bücher abgesetzt werden, weil die Gemeinden dafür weniger Mittel bereitstellen, kann ein verlag es sich nicht mehr leisten, in einem größeren Bundesland ein Buch nicht genehmigt zu bekommen. Das heibt dann z.B., daß eine Deutschredaktion kurz davor war, in letzter Minute in ihrem neuen Sprachbuch das Wort "Substantiv" in "Nomen" zu ändern, weil man vermutete, damit den in Uberarbeitung befindlichen Lehrplänen gerechter $z u$ werden. Man unterlieB diese Änderung, zum Glück für den Markt in BadenWürttemberg. Zur zeit muB noch abgewartet werden, ob neben der Substantiv-Ausgabe eine Nomen-Ausgabe für andere Bundesländer nötig werden wird. An diesem Punkt wird wohl die absolute Lächerlichkeit terminologischer Festschreibungen im Lehrplan deutlich. Je mehr solcher setzungen die Bundesländer einzeln vornehmen, desto mehr reduziert sich die Entscheidung über Lehrbücher auf den nur terminologischen Gesichtspunkt und schliebt konzeptionelle Uberlegungen aus. Dazu kommt dann noch die unterschiedliche Verteilung des grammatischen stoffes über die Schuljahre. Es sieht so aus, als würden die schulbuchverlage in $\mathrm{zu-}$ kunft doch wieder - wo nicht zu Regionalausgaben für Einzelländer, so doch zu parallelausgaben - mit unterschiedlichen Termini und unterschiedlicher stoffverteilung gezwungen. Was dann auch bedeutet, daß nur noch große verlage mithalten können - auch auf dem Wege der Lehrplanhoheit der Bundesländer kann die Schulbuchvielfalt reduziert werden. 